\title{
A Macroeconomic Model for Resource Allocation in Large-Scale Distributed Systems
}

\author{
Xin Bai ${ }^{\text {a }}$, Dan C. Marinescu ${ }^{\text {a,* }}$, Ladislau Bölöni ${ }^{\text {a }}$, \\ Howard Jay Siegel $^{\mathrm{b}}$, Rose A. Daley ${ }^{\mathrm{c}}$, I-Jeng Wang ${ }^{\mathrm{c}}$

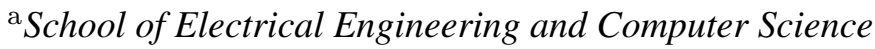 \\ University of Central Florida, Orlando, FL 32816-2362 \\ ${ }^{\mathrm{b}}$ Department of Electrical and Computer Engineering \\ and Department of Computer Science \\ Colorado State University, Fort Collins, CO 80523-1373 \\ ${ }^{\mathrm{c}}$ Applied Physics Laboratory
}

Johns Hopkins University, 11100 Johns Hopkins Road Laurel, MD 20723-6099 


\begin{abstract}
In this paper we discuss an economic model for resource sharing in large-scale distributed systems. The model captures traditional concepts such as consumer satisfaction and provider revenues and enables us to analyze the effect of different pricing strategies upon measures of performance important for the consumers and the providers. We show that given a particular set of model parameters the satisfaction reaches an optimum; this value represents the perfect balance between the utility and the price paid for resources. Our results confirm that brokers play a very important role and can influence positively the market. We also show that consumer satisfaction does not track the consumer utility, these two important performance measures for consumers behave differently under different pricing strategies. Pricing strategies also affect the revenues obtained by providers, as well as, the ability to satisfy a larger population of users.
\end{abstract}

Key words: Resource Allocation, Macroeconomic Model, Utility, Price, Consumer Utility, Consumer Satisfaction, Large-Scale Distributed System

* Corresponding author. Phone: (407)8234860 FAX: (407)8235419

Email address: dcmecs . udf . edu (Dan C. Marinescu). 


\section{Introduction and Motivation}

Computational, service, and data grids, peer-to-peer systems, and ad-hoc wireless networks are examples of open systems. Individual members of the community contribute computing cycles, storage, services, and communication bandwidth to the pool of resources available to the entire community; resources as well as consumers of resources could belong to different administrative domains. In this case it is difficult to devise global resource allocation policies and there is no central authority to enforce global policies and schedules. The existence of multiple administrative domains is a reality in the Grid environments and the Internet. In the context of our research, each administrative domain corresponds to a different organization and has complete control of its resources and dictates the price and the amount of resources available. A broker mediates between producers and consumers in different administrative domains. The research reported in this paper investigates the use of macroeconomic models for resource allocation in heterogeneous, distributed computing and communication systems.

Market-oriented economies have proved their advantages over alternative means to control and manage resource allocation in social systems [8]. It seems reasonable to adapt some of the successful ideas of economical models to resource allocation in large-scale computing systems and to study market-oriented resource allocation algorithms. As shown by recent studies, economic models are attractive for resource providers, beneficial for the consumers of resources, and have societal benefits for large-scale distributed systems [41-43]. Fewer resources are wasted, while excess capacity and overloading are averaged over a very large number of providers and consumers. The system is more scalable and decision-making is distributed. In an economic model, all participants are considered self-interested. The resource providers are trying to maximize their revenues. The consumers want to obtain the maximum possible resources for the minimum possible price. The large number of participants makes one-to-one negotiations expensive and unproductive. 
In 1933 the Norwegian economist and Nobel laureate Ragnar Frisch introduced the dichotomy macroeconomy/microeconomy [16]. Macroeconomics deals with the economy as a whole and studies aggregate trends such as total consumption and production [36], while microeconomics is primarily focused on the economic behavior of individual units and the role of prices in allocation of scarce resources [39]. While in our model we simulate the individual customers and producers, the objectives are to improve the aggregate utility and satisfaction of the entire user population and all the resources of the distributed system. Thus, it seems more appropriate to call it a macroeconomic model.

The model presented in this paper is based upon concepts borrowed from economics, such as utility and consumer satisfaction. Informally, utility quantifies the benefits obtained as the result of being granted a certain amount of resources. Utility-based resource allocation models have proved their potential in a different context, e.g., when the only resource is the radio bandwidth, the size of the population is limited, and each participant has a unique role (e.g., is a consumer) [4]. The heterogeneity of a large-scale distributed system, the large spectrum of resources and demands placed upon these resources, the scale of the system, the autonomy of individual resource providers, and the dual role of individual actors, as consumer of some resources and provider for others, add complexity to the models we study in this paper.

Different utility functions can be considered; a utility function should be: (i) monotonically increasing with the amount of allocated resource; (ii) convex for high resource values; and (iii) fast growing for small amounts of allocated resources. Increasing the resource allocation will yield lower and lower increase in utility. Intuitively, this is justified by Amdalh's law: once a resource is plentiful, the performance bottleneck moves to another type of resource, and adding additional resource yields little benefit. These observations lead us to conclude that the utility function will have an S-shaped, sigmoid curve. Sigmoid functions have all the desirable properties and have been used in many economic models, in biology, and some 
other areas, as we discuss in Section 3.

In our model, each resource is characterized by a vector with several components. In the general case, a request may require multiple resources or resources with multiple attributes thus, more general utility functions are surfaces in hyperspaces with several dimensions. A discussion of the relative advantages of different families of utility functions is an important and timely subject, but beyond the scope of this paper. While the sigmoid is selected for us here, it could be replaced by other functions within the framework of our model.

We define a measure of consumer's satisfaction that takes into account the utility resulting from resource consumption and the price paid by the consumer. We show that given a particular set of model parameters the satisfaction reaches an optimum; this value represents the perfect balance between the utility and the price paid for resources.

Consumer satisfaction is a more general metric than QoS that typically refers to a single performance measure (e.g., time to completion or end-to-end delay), and does not reflect pricing. Moreover, QoS requirements are generally specified by an upper bound (e.g., the jitter should be less than $m$ milliseconds), while one can provide a continuous function describing the utility and select a provider that optimizes the satisfaction value, or one that is very close to the optimum.

We also show that consumer satisfaction does not track the consumer utility; these two important performance measures for consumers behave differently under different pricing strategies. Pricing strategies also affect the revenues obtained by providers, as well as the request acceptance ratio. We introduce three pricing policies and investigate the effect of several parameters upon critical measures of performance for producers and consumers. The pricing policies are affected by the relationship between the amount of resources required and the total amount we pay for them, as well as the overall state of the system. We analyze the case when the price per unit is constant regardless of the amount of resources consumed (linear 
pricing); pricing to encourage consumption, i.e., the more resources are used the lower becomes the average unit price (sub-linear pricing); and pricing to discourage consumption, i.e., the more resources are used the higher becomes the average unit price (super-linear pricing). We also analyze the effect of resource abundance upon pricing strategies.

We foresee an adaptive and intelligent user behavior based upon the idea that, in the general case, the same goal may be achieved through different means. We suggest the transformation of imperative requests into elastic ones that reflect the level of utility. Practically, we specify a range of target systems, e.g, clusters with 100 to 10,000 nodes, and for each potential target of an elastic request we compute a satisfaction value and choose the one leading to the largest satisfaction. Assume that we process a very large number of images and could use a variety of system configurations each with its own utility and the corresponding satisfaction value. Our best option is to use a cluster with 10,000 nodes with 4 GB of memory per node, but there are only a few such systems; at the other extreme, we could use a cluster more likely to be available soon with only 100 nodes and 2 GB of main memory per node, but the execution time would increase by four orders of magnitude. An elastic request quantifies the urgency of the request and allows the broker and/or the user to compare the satisfaction values and decide whether to pay a higher price for a unique resource, or use a readily available one.

Several models including ours include middlemen to mediate access to resources a strategy rather common for agent systems. The role of a broker is to reconcile the selfish objectives of individual resource providers and consumers with some global, societal objectives, e.g., to maximize the resource utilization of the system.

The best analogy for a broker in our model is a financial advisor in the real world. Clients trust their financial advisors and disclose their financial objectives (corresponding to the utility and satisfaction functions) to them. At the same time, the clients know and accept the fact that the financial advisor will act in the context of existing laws and stock market 
regulations, thus, it also serves societal objectives.

Most macroeconomic models include policy makers that establish societal objectives. For example the Federal Reserve Board establishes monetary policies in the US and there are counterparts in other countries. In a global economy the policy makers sometimes coordinate their behavior. In the future our simulation studies should be extended to include policy makers and multiple brokers.

Our results confirm that brokers play a very important role and can influence positively the market. The development of broker-to-broker coordination models and an analysis of a more complex system is well beyond the scope of this paper due to the complexity of the analysis and space limitations. As expected, even with a set of simplifying assumptions, the models are extremely complex and can only be evaluated through simulation.

The contributions of this paper are: (i) a macroeconomic model that includes policy makers whose role is to establish societal objectives, trusted middleman whose role is to ensure maximum satisfaction to their clients, and producers and consumers of resources; (ii) utility and satisfaction functions, as well as pricing policies; (iii) a simulation study of the behavior of a system with a single broker.

The paper is organized as follows: we survey different economic models applied to information systems in Section 2 and compare their features with our model. In Section 3, we introduce the basic elements of our model and define utility and satisfaction function, as well as pricing strategies. The role of the middlemen is discussed in Section 4 and the results of a simulation study are analyzed in Section 5; finally, we present our conclusions in Section 6. 


\section{Related Work}

The development of the first global macroeconomic model, the Wharton Econometric Forecasting Associates LINK project, started in 1968 under the leadership of Nobel laureate Lawrence Klein [30]. There are two basic analytical approaches to classical macroeconomics: (a) Keynesian economics focused on demand and (b) supply-side economics focused on supply.

Interestingly enough, the most complex information system ever conceived, the Internet, takes advantage of ideas that can be traced back to macroeconomics. While the Internet is based upon a best effort service model, supply-side economics are reflected by overprovisioning, namely building an excess bandwidth to ensure some levels of QoS.

Frank Kelly developed in late 1990s an analytical model for a self-managed Internet based upon utility and cost [25]. Kelly considers a set of sessions $s \in S$ that use a set of links $l \in L$; each link $l$ has a capacity $C$. If $L_{s}$ is the set of links used by session $s$, then $U_{s}\left(r_{s}\right), S \in$ $L_{s)}$ is a strictly concave, increasing function of the packet source rate, $r_{s}$. He attempts to maximize system utility with the constraint that the total bandwidth used on each link by all connections is lower than the link capacity. If $p_{s}$ is the price function of the rate, then a distributed algorithm solves a greedy optimization problem for every session:

$$
\max U_{s}\left(r_{s}\right)-p_{s} r_{s}
$$

It turns out that the adaptive congestion control mechanism introduced in early 1980s for TCP can be well described by Kelly's model developed in late 1990s; the price in this case is the probability of losing packets and the utility is a simple function of the round trip time (RTT). 
Extending Kelly's model to a large-scale distributed system consisting of a collection of heterogeneous systems, while feasible, would be rather impractical; the need to differentiate services for individual consumers and specify a different utility function for each one of them, the variety of prices and the fact that individual entities are consumers and providers at the same time, makes such an extension very hard and possibly infeasible computationally.

Even though theoretical studies of economic models applied to information systems are only now beginning to emerge, several companies including IBM (E-Business On Demand [23]), HP (Adaptive Enterprise [22]), Sun Microsystems (pay-as-you-go [38]), as well as startups such as Entropia, ProcessTree, Popular Power, Mojo Nation, United Devices, and Parabon are embedding economics into their resource allocation systems. Economic concepts and ideas are used for distributed storage systems such as the Stanford Peers Initiative [14] and GnuNet [19] and distributed databases [3,37]. Java Market [2], JaWS [26], Xenoservers [31], and others apply economic models for computer services.

The economic concepts and strategies embedded in existing or proposed systems and models [11] are summarized in Table 1 and surveyed below. An auction starts with owners announcing resources and inviting bids; consumers bid and the winner gets access to the resource. In an English auction, when no bidder is willing to increase the bid, the auction ends and the highest bidder wins; in first-price sealed-bid auction, every bidder submits a sealed-bid and the highest bidder wins; in a Vickrey auction, every bidder submits a sealed-bid and the highest bidder wins at the price of the second highest bidder; a Dutch auction starts with a high price lowered until a bidder is willing to pay the current price. In bid-based proportional resource sharing the percentage of resources allocated is a function of user's bid relative to others. Bargaining requires direct negotiations between producers and consumers until they reach a mutually agreeable price. Bartering is conducted among the members of a community that share each other's resources. In commodity markets, providers advertise their resource prices and charge users based on the amount of resources used; posted price 
Table 1

Economic concepts and strategies in different systems. Abbreviations: Au - Auction; Ba - Bargaining; Bt - Bartering; Cm - Commodity market; Co - Coalition; Mo - Monopoly; Ut -Utility; Cs Consumer satisfaction; Pr - Pricing policy; Results - performance results reported in literature, Analitical/Simulation.

\begin{tabular}{|l|cccccccccc|}
\hline System & Au & Ba & Bt & Cm & Co & Mo & Ut & Cs & Pr & Results \\
\hline ContractNet [35] & Yes & Yes & No & Yes & No & No & No & No & No & Deployed \\
Condor [13] & No & No & No & Yes & No & No & No & No & Yes & Deployed \\
Enhanced MOSIX [1] & No & No & No & No & No & No & Yes & No & Yes & Simulated \\
Mariposa [27] (based on & Yes & Yes & No & Yes & No & No & No & No & No & Prototype \\
the Contract Net) & & & & & & & & & & \\
Rexec/Anemone [12] & No & No & No & Yes & No & Yes & No & No & Yes & Prototype \\
SETI@ home [32] & No & No & No & Yes & No & No & No & No & No & Deployed \\
Spawn [41] & Yes & No & No & No & No & No & Yes & No & Yes & Prototype \\
Sun [38]) & No & No & No & Yes & No & No & No & No & No & Deployed \\
Our model & No & No & No & No & No & No & Yes & Yes & Yes & Simulation \\
\hline
\end{tabular}

allows providers to advertise special offers to attract consumers. In case of a monopoly, one or a small number of resource providers decide a non-negotiable the price. Pricing policy could be based on a flat fee, the resource usage duration, the subscription, the demand and supply [28], or could be designed to encourage or discourage consumption.

Arguments that commodities markets are better choices for controlling grid resources than auction strategies are presented in $[42,43]$ based upon concepts such as price stability, market equilibrium, consumer efficiency, and provider efficiency. An approach to implement automatic selection of multiple negotiation models to adapt to the computation needs and changes in a resource environment is discussed in [33]. A task-oriented mechanism for measuring the economic value of using heterogeneous resources as a common currency is analyzed in [20]; resource consumers can compare the advantage of participating in a computational grid with 
the alternative of purchasing their own resources necessary, and resource providers can evaluate the profit of putting their resources into a grid. A comparative analysis of market-based resource allocation by continuous double auctions and by the proportional share protocol versus a conventional round-robin approach is presented in [18]. A game theoretic pricing strategy for efficient job allocation in mobile grids is discussed in [17]; a grid resource allocation model based upon a game theoretic approach is presented in [24].

Table 1 summarizes the key features of a variety of economic-based systems for resource allocation. Our approach differs from other models in that it is based upon utility and satisfaction functions and pricing policies.

\section{Basic Concepts}

An efficient and fair utilization of the resources can be obtained only through a scheme that gives incentives to the providers to share their resources and that encourages the consumers to maximize the utility of the received resources. A well-tested model for such a scheme is based on an economic model, in which the resources need to be paid for in a real or virtual currency. This model has the advantage of being provably scalable, and we can successfully reuse or adapt the models that govern the economy in our society.

To study possible resource management policies, we have to develop resource consumption models that take into account different, possibly contradictory, views of the benefits associated with resource consumption as well as the rewards for providing resources to the consumer population. Such models tend to be very complex and only seldom amenable to analytical solutions.

In this section we introduce the basic concepts and notations for our model. First, we introduce price, utility, and satisfaction functions; then we present our resource provider- 
consumer model. To capture the objectives of the entities involved in the computational economy we use: (i) a consumer utility function, $0 \leq u(r) \leq 1$, to represent the utility provided to an individual consumer, where $r$ represents the amount of allocated resource; (ii) a provider price function, $p(r)$, imposed by a resource provider, and (iii) a consumer satisfaction function, $s(u(r), p(r)), 0 \leq s \leq 1$, to quantify the level of satisfaction; the satisfaction depends on both the provided utility and the paid price.

\subsection{Price Functions}

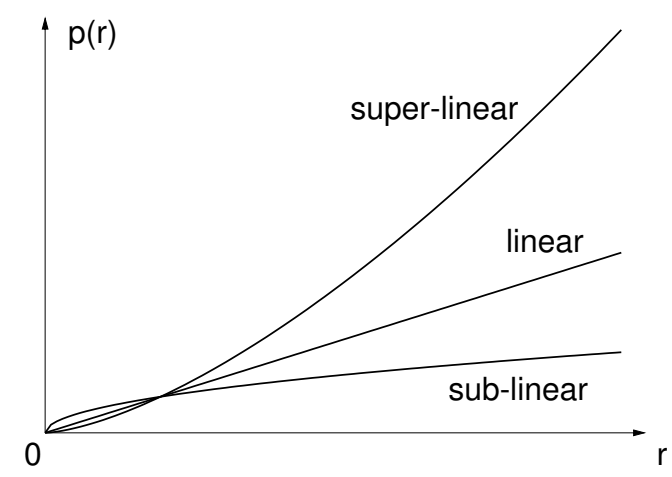

(a)

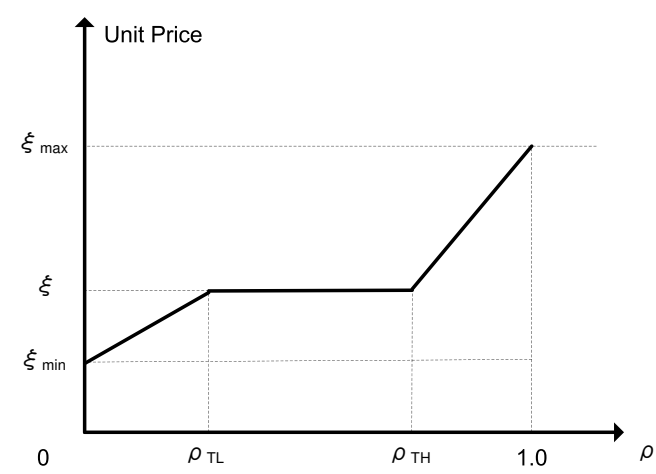

(b)

Fig. 1. (a) Sub-linear, linear, and super-linear price functions. (b) The unit price varies with $\rho$, the load index of the provider.

We discuss the three pricing functions in Figure 1(a). Given the constant, $\xi$, the three particular pricing functions we choose are:

(a) The price per unit is constant regardless of the amount of resources consumed (linear pricing):

$$
p(r)=\xi \cdot r
$$

(b) Discourage consumption: the more resources are used, the higher becomes the average 
unit price (super-linear pricing):

$$
p(r)=\xi \cdot r^{d}
$$

where $d>1$. For this equation, we use $d=1.5$ throughout the remainder of the paper.

(c) Encourage consumption: the more resources are used, the lower becomes the average unit price (sub-linear pricing):

$$
p(r)=\xi \cdot r^{e}
$$

where $e<1$. For this equation, we use $e=0.5$ throughout the remainder of the paper.

We also analyze the effect of resource abundance; in this case we define the load index $\rho$ as the ratio of total amount of allocated resources to the capacity of the provider and consider three regions: low, medium, and high load index. We denote the low, medium, and high regions as the interval of $\left[0, \rho_{T L}\right),\left[\rho_{T L}, \rho_{T H}\right]$, and $\left(\rho_{T H}, 1\right]$, respectively, as shown in Figure 1(b). The pricing strategy for each region is different. We consider two models, EDL Encourage/Discourage Linear, and EDN - Encourage/Discourage Nonlinear. The choice of the $\rho_{T L}, \rho_{T H}$ is basically a policy decision. However, in order to have the desired influence on the system as a whole, the three intervals need to be of a sufficient size. Values such as $\rho_{T L}=0.49$ and $\rho_{T H}=0.51$ make the target interval unreasonably small; very low $\rho_{T L}$ and very high $\rho_{T H}$ values make the pricing strategy degenerate into a constant price strategy. The values used throughout this paper are $\rho_{T L}=0.3$ and $\rho_{T H}=0.7$.

For the first model, the unit price is constant in each region, but different in different regions, as defined in Equation 4, and shown in Figure 1(b). We introduce three prices, each corresponding to a range of the system load: minimal, $\xi_{\min }$, maximal, $\xi_{\max }$, and $\xi$, the price corresponding to medium load. For low load the providers use lower prices to encourage resource consumption, but do not lower the price below $\xi_{m i n}$. For high load, the providers gradually increase the price, up to $\xi_{\max }$. The choice of the $\xi_{\min }$ and $\xi_{\max }$ are a matter of policy, however, too low values for $\xi_{\min }$ would make resources basically free for nodes with 
low utilization, and very high values of $\xi_{\max }$ would make resources too expensive. We used the values of $\xi_{\max }=2 \times \xi$, and $\xi_{\min }=0.5 \times \xi$ throughout the remainder of the paper.

$$
p(r)= \begin{cases}\left(\xi_{\min }+\frac{\rho}{\rho_{T L}}\left(\xi-\xi_{\min }\right)\right) \cdot r & \text { if } \rho \in\left[0, \rho_{T L}\right) ; \\ \xi \cdot r & \text { if } \rho \in\left[\rho_{T L}, \rho_{T H}\right] \\ \left(\xi+\frac{\rho-\rho_{T H}}{1.0-\rho_{T H}}\left(\xi_{\max }-\xi\right)\right) \cdot r & \text { if } \rho \in\left(\rho_{T H}, 1.0\right] .\end{cases}
$$

For the second model, when $\rho$ is low, the provider uses a sub-linear price function; when $\rho$ is high, the provider uses a super-linear price function; otherwise, the provider uses a linear price function, as expressed by Equation 5:

$$
p(r)=\left\{\begin{array}{l}
\xi \cdot r^{e} \text { if } \rho \in\left[0, \rho_{T L}\right) \\
\xi \cdot r \text { if } \rho \in\left[\rho_{T L}, \rho_{T H}\right] \\
\xi \cdot r^{d} \text { if } \rho \in\left(\rho_{T H}, 1.0\right]
\end{array}\right.
$$

where $e<1$ and $d>1$. The choice of $e$ and $d$ follow similar considerations like the choice of parameters for the EDL model: we need to encourage and discourage the customers, while still maintaining the prices in a justifiable range. In this paper we are using the values of $e=0.5$ and $d=1.5$, which provide an appropriate range of prices.

\subsection{Utility Function}

The utility function should be a non-decreasing function of $r$, i.e., we assume that the more resources are allocated to the consumer, the higher the consumer utility is. However, when enough resources have been allocated to the consumer, i.e., some threshold is reached, an increase of allocated resources would bring no improvement of the utility. On the other hand, 
if the amount of resources is below some threshold the utility is extremely low. Thus, we expect the utility to be a concave function and reach saturation as the consumer gets all the resources it can use effectively. These conditions are reflected by the following equations:

$$
\frac{d u(r)}{d r} \geq 0, \quad \lim _{r \rightarrow \infty} \frac{d u(r)}{d r}=0
$$

For example, if a parallel application could use at most 100 nodes of a cluster, its utility reflected by a utility function does not increase if its allocation increases from 100 to 110 nodes. If we allocate less than 10 nodes then the system may spend most of its time paging and experiencing cache misses and the execution time would be prohibitively high.

Different functions can be used to model this behavior and we choose one of them, a sigmoid:

$$
u(r)=\frac{(r / \omega)^{\zeta}}{1+(r / \omega)^{\zeta}}
$$

where $\zeta$ and $\omega$ are constants provided by the consumer, $\zeta \geq 2$, and $\omega>0$. Clearly, $0 \leq$ $u(r)<1$ and $u(\omega)=1 / 2$.

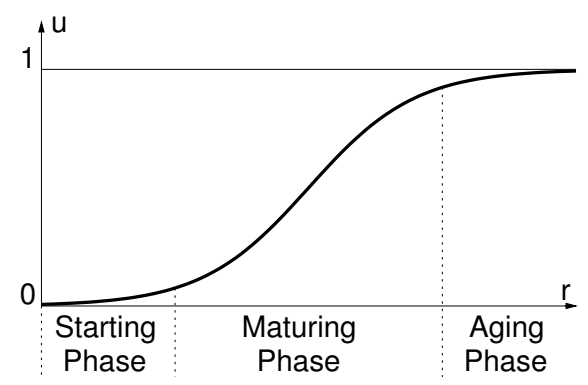

(a)

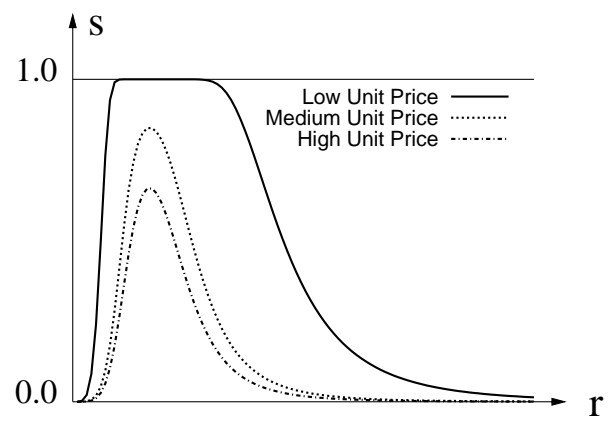

(b)

Fig. 2. (a) A sigmoid is used to model the utility function; a sigmoid includes three phases: the starting phase, the maturing phase, and the aging phase. (b) The satisfaction function for a sigmoid utility function and three linear price functions with low, medium, and high unit price.

A sigmoid is a tilted S-shaped curve that could be used to represent the life-cycles of living, 
as well as man-made, social, or economical systems. It has three distinct phases: an incipient or starting phase, a maturing phase, and a declining or aging phase, as shown in Figure 2(a).

\subsection{Satisfaction Function}

A consumer satisfaction function takes into account both the utility provided to the consumer and the price paid for the resources. For a given utility, the satisfaction function should increase when the price decreases and, for a given price, the satisfaction function should increase when the utility $u$ increases. These requirements are reflected by Equation (8).

$$
\frac{\partial s}{\partial p} \leq 0, \quad \frac{\partial s}{\partial u} \geq 0
$$

Furthermore, a normalized satisfaction function should satisfy the following conditions:

- the degree of satisfaction, $s(u(r), p(r))$, for a given price $p(r)$, approaches the minimum, 0 , when the utility, $u(r)$, approaches 0 ;

- the degree of satisfaction, $s(u(r), p(r))$, for a given price $p(r)$, approaches the maximum, 1, when the utility, $u(r)$, approaches infinity;

- the degree of satisfaction, $s(u(r), p(r))$, for a given utility $u(r)$, approaches the maximum, 1 , when the price, $p(r)$, approaches 0 ; and

- the degree of satisfaction, $s(u(r), p(r))$, for a given utility $u(r)$, approaches the minimum, 0 , when the price, $p(r)$, approaches infinity.

These requirements are reflected by Equations (9) and (10).

$$
\begin{aligned}
& \forall p>0, \lim _{u \rightarrow 0} s(u, p)=0, \quad \lim _{u \rightarrow \infty} s(u, p)=1 \\
& \forall u>0, \lim _{p \rightarrow 0} s(u, p)=1, \quad \lim _{p \rightarrow \infty} s(u, p)=0
\end{aligned}
$$


A candidate satisfaction function is [39]:

$$
s(u, p)=1-e^{-\kappa \cdot u^{\mu} \cdot p^{-\epsilon}}
$$

where $\kappa, \mu$, and $\epsilon$ are appropriate positive constants. The satisfaction function based upon the utility function in Equation 7 is normalized; given a reference price $\phi$ we consider also a normalized price function and we end up with a satisfaction function given by:

$$
s(u, p)=1-e^{-\kappa \cdot u^{\mu} \cdot(p / \phi)^{-\epsilon}} .
$$

Because $u$ and $p$ are functions of $r$, satisfaction increases as more resources are allocated, reaches an optimum, and then declines, as shown in Figure 2(b). The optimum satisfaction depends upon the pricing strategy; not unexpectedly, the higher the unit price, the lower the satisfaction.

The $3 D$ surfaces representing the relationship $s=s(r, \xi)$ between satisfaction $s$ and the unit price $\xi$ and amount of resources $r$ for several pricing functions (super-linear, linear, and sublinear) are presented in Figure 3. As we can see from the cut through the surfaces $s=s(r, \xi)$ at a constat $\xi$ when we discourage consumption (super-linear pricing) the optimum satisfaction is lower and occurs for fewer resources; when we encourage consumption (sub-linear pricing) the optimum satisfaction is improved and occurs for a larger amount of resources. These plots reassure us that the satisfaction function has the desired behavior.

\subsection{Resource Provider-Consumer Model}

Consider a system with $n$ providers offering computing resources and $m$ consumers. To simplifying the model, we assume that the two sets are disjoint. Call $\mathcal{U}$ the set of consumers and $\mathcal{R}$ the set of providers. The $n$ providers are labeled 1 to $n$ and the $m$ consumers are 


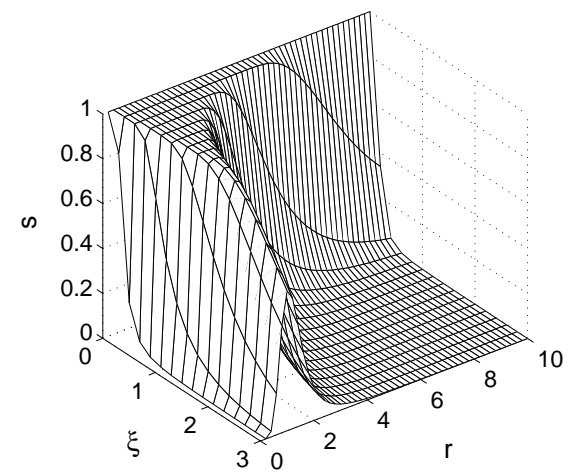

(a)

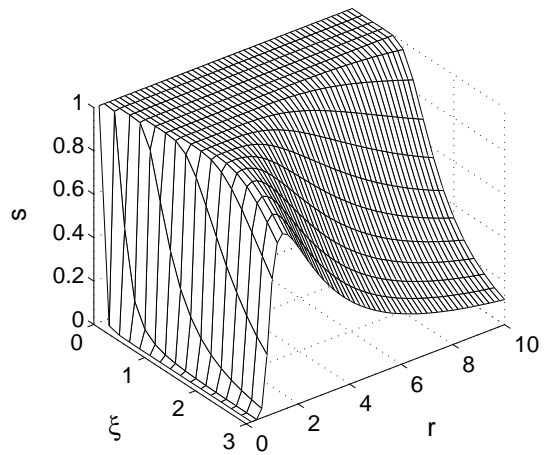

(c)

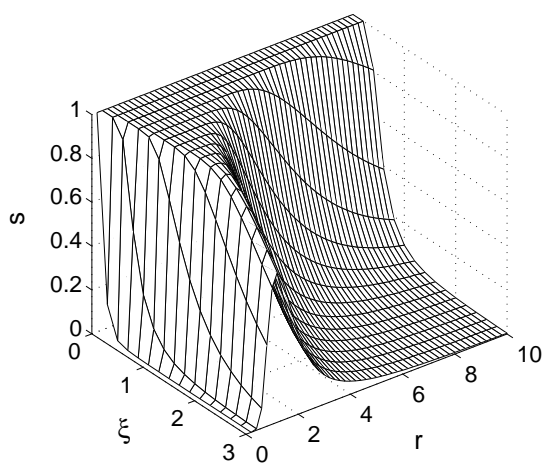

(b)

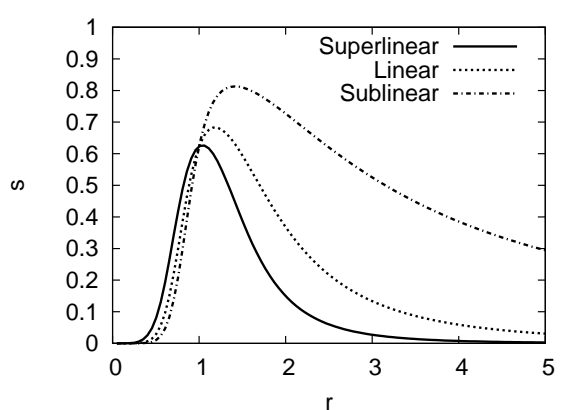

(d)

Fig. 3. The relationship between satisfaction $s$ and the unit price $\xi$ and amount of resources $r$. The satisfaction function is based on a sigmoid utility function and different price functions: (a) discourage consumption (super-linear); (b) linear; (c) encourage consumption (sub-linear); (d) a cut through the three surfaces at a constant $\xi$.

labeled 1 to $m$. Consider provider $R_{j}, 1 \leq j \leq n$, and consumer $U_{i}, 1 \leq i \leq m$, that could potentially use resources of that provider.

Let $r_{i j}$ denote the resource (defined below) of $R_{j}$ allocated to consumer $U_{i}$ and let $u_{i j}$ denote its utility for consumer $U_{i}$. Let $p_{i j}$ denote the price paid by $U_{i}$ to provider $R_{j}$. Let $t_{i j}$ denote the time $U_{i}$ uses the resource provided by $R_{j}$. Let $c_{j}$ denote the resource capacity of $R_{j}$, i.e., the amount of resources regulated by $R_{j}$. 
The term "resource" here means a vector with components indicating the actual amount of each type of resource:

$$
r_{i j}=\left(r_{i j}^{1} r_{i j}^{2} \ldots r_{i j}^{l}\right)
$$

where $l$ is a positive integer and $r_{i j}^{k}$ corresponds to the amount of resource of the $k$-th type. The structure of $r_{i j}$ may reflect the rate of CPU cycles, the physical memory required by the application, the secondary storage, the number of nodes and the interconnection bandwidth (for a multiprocessor system or a cluster), the network bandwidth (required to transfer data to/from the site), the graphics capabilities, and so on.

The utility of resource of the $k$-th type provided by $R_{j}$ for consumer $U_{i}$ is a sigmoid:

$$
u_{i j}^{k}=u\left(r_{i j}^{k}\right)=\frac{\left(r_{i j}^{k} / \omega_{i}^{k}\right)^{\zeta_{i}^{k}}}{1+\left(r_{i j}^{k} / \omega_{i}^{k}\right)^{\zeta_{i}^{k}}}
$$

where $\zeta_{i}^{k}$ and $\omega_{i}^{k}$ are constants provided by consumer $U_{i}, \zeta_{i}^{k} \geq 2$, and $\omega_{i}^{k}>0$. Clearly, $0<u\left(r_{i j}^{k}\right)<1$ and $u\left(\omega_{i}^{k}\right)=1 / 2$.

The overall utility of resources provided by $R_{j}$ to $U_{i}$ is:

- the product over the set of resources provided by $R_{j}$, i.e., $u_{i j}=\prod_{k=1}^{l} u_{i j}^{k}$, or

- the weighted average over the set of resources provided by $R_{j}$, i.e., $u_{i j}=\frac{1}{l} \sum_{k=1}^{l} a_{i j}^{k} u_{i j}^{k}$, where $a_{i j}^{k}$ values are provided by consumer $U_{i}$ and $\sum_{k=1}^{l} a_{i j}^{k}=1$.

Let $p_{i j}^{k}$ denote the price consumer $U_{i}$ pays to provider $R_{j}$ for a resource of type $k$. The total price for consumer $U_{i}$ for resources provided by provider $R_{j}$ is:

$$
p_{i j}=\sum_{k=1}^{l} p_{i j}^{k}
$$

The total cost for consumer $U_{i}$ for resources provided by provider $R_{j}$ is $p_{i j} \times t_{i j}$.

Based on Equation 12, we define the degree of satisfaction of $U_{i}$ for a resource of the $k$-th 
type provided by provider $R_{j}$ as:

$$
s_{i j}^{k}\left(u_{i j}^{k}, p_{i j}^{k}\right)=1-e^{-\kappa_{i}^{k} u_{i j}^{k} \mu_{i j}^{k}}\left(p_{i j}^{k} / \phi_{i}^{k}\right)^{-\epsilon_{i}^{k}}, \quad \kappa_{i}^{k}, \phi_{i}^{k}, \mu_{i}^{k}, \epsilon_{i}^{k}>0
$$

where $\kappa_{i}^{k}, \mu_{i}^{k}$ and $\epsilon_{i}^{k}$ are appropriate positive constants and $\phi_{i}^{k}$ is a reference price.

The overall satisfaction of consumer $U_{i}$ for resources provided by $R_{j}$ is:

- the product over the set of resources provided by $R_{j}$, i.e., $s_{i j}=\prod_{k=1}^{l} s_{i j}^{k}$, or

- the weighted average over the set of resources provided by $R_{j}$, i.e., $s_{i j}=\frac{1}{l} \sum_{k=1}^{l} b_{i j}^{k} s_{i j}^{k}$, where $b_{i j}^{k}$ values are provided by consumer $U_{i}$ and $\sum_{k=1}^{l} b_{i j}^{k}=1$.
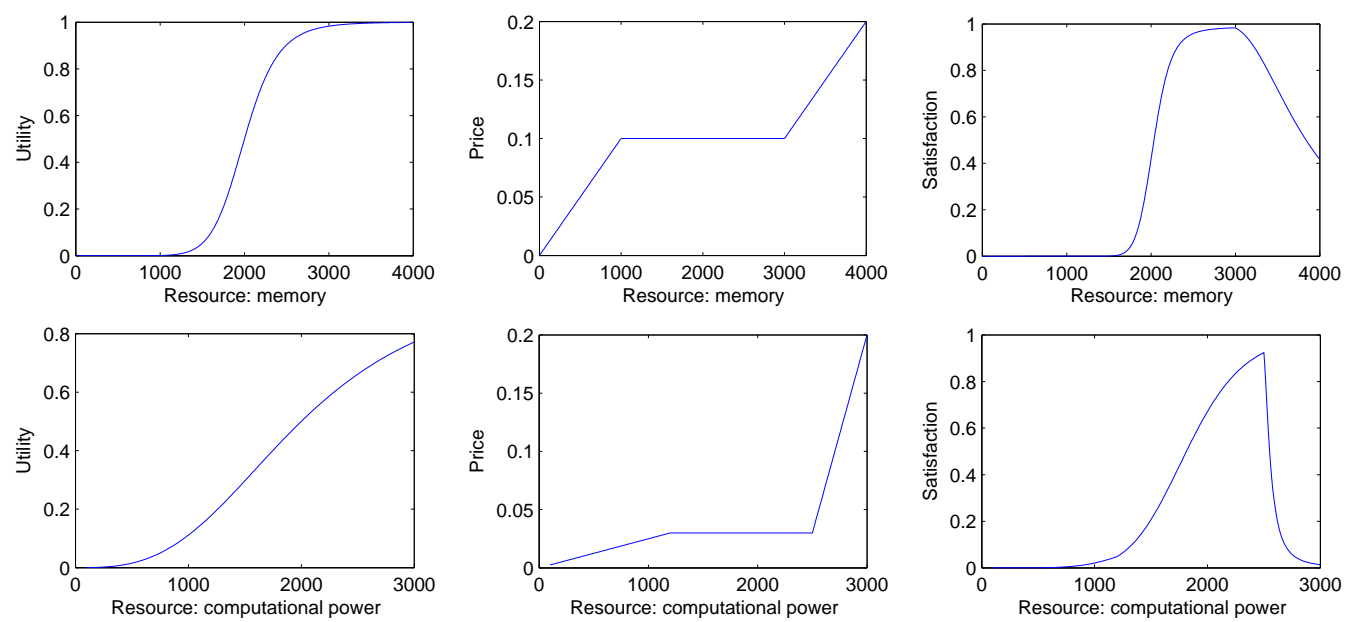

Fig. 4. Example of utility (left), price (middle) and satisfaction (right) curves for two resources: memory (upper row) and computational power (lower row).

We conclude this section with an example. Let us consider a system which has two resources: memory (measured in MBytes) and computational power (measured in MHz). Let us consider a client which has its utility function calculated by setting $\omega_{m}=2000, \zeta_{m}=10$ for the memory, while for the computational power $\omega_{c p}=2000$ and $\zeta_{c p}=3$. The shape of the utility curve is shown in Figure 4, left. The utility curve is almost linear for the computational power, while it has a steep ramp for memory at the value of around 2000 MBytes. A memory allocation smaller than 1000 MBytes has virtually no utility, while adding extra memory above 3000 MBytes yields very little benefit. The price curves are shown in Fig- 
ure 4, middle. The shapes of the curves are justified by objective considerations: for instance high performance processors are an order of magnitude more expensive than consumer grade processors. Finally, the satisfaction function is calculated with the values for the memory being: $\kappa_{m}=0.02, \phi_{m}=0.6, \mu_{m}=3$ and $\epsilon_{m}=3$, while for computational power $\kappa_{c p}=0.03$, $\phi_{c p}=0.2, \mu_{c p}=3$ and $\epsilon_{c p}=3$. The satisfaction curves are shown in Figure 4, right. We will assume that the overall satisfaction is the product of the satisfaction for memory and computational power.

Let us now consider the case when the customer receives four offers A, B, C and D. The offers and the associated cost and satisfaction values are summarized below:

\begin{tabular}{|l|l|l|l|l|l|l|}
\hline & Memory & C. power & Cost & Cost & Total cost & Satisfaction \\
\hline A & 3000 & 2000 & 300 & 60 & 360 & 0.6676 \\
B & 2500 & 2500 & 250 & 83.5 & 333.5 & 0.8132 \\
C & 2300 & 2500 & 230 & 83.5 & 313.5 & 0.7574 \\
\hline
\end{tabular}

Under these conditions, the user will choose offer B, which offers the highest satisfaction. Notice that B is neither the offer with the largest amount of resources, neither the cheapest offer.

\section{The Role of Brokers}

In this paper, we concentrate on optimal resource management policies. A policy is optimal when the satisfaction function, which reflects both the price paid to carry out a task and the 
utility resulting from the completion of the task, reaches a maximum. A broker attempts to operate at or near this optimum.

The role of a broker is to mitigate access to resources. In this paper, we consider providerbroker-consumer models that involve the set of resource providers $\mathcal{R}$, the set of consumers $\mathcal{U}$, and broker $B$. These models assume that a consumer must get all of its resources from a single provider. Brokers have "societal goals" and attempt to maximize the average utility and revenue, as opposed to providers and consumers that have individualistic goals; each provider wishes to maximize its revenue, while each consumer wishes to maximize its utility and do so for as the lowest cost possible. To reconcile the requirements of a consumer and the candidate providers, a broker chooses a subset of providers such that the satisfaction is above a high threshold and all providers in the subset have equal chances to be chosen by the consumer. We call the size of this subset satisficing size, and denote it by $\sigma$; the word "satisfice" was coined by Nobel Prize winner Herbert Simon in 1957 to describe the desire to achieve a minimal value of a variable instead of its maximum [34].

The resource negotiation protocol consists of the following steps:

(1) All providers reveal their capacity and pricing parameters to the broker: $\forall R_{j} \in \mathcal{R}$ send vectors $c_{j}$ and $\xi_{j}$ where each element corresponds to one type of resource.

(2) A consumer $U_{i}$ sends to the broker a request with the following information:

(a) the parameters of its utility function: vectors $\zeta_{i}$ and $\omega_{i}$ where each element corresponds to one type of resource,

(b) the parameters of its satisfaction function: vectors $\mu_{i}, \epsilon_{i}, \kappa_{i}$ and $\phi_{i}$ where each element corresponds to one type of resource, and

(c) the number of candidate resource providers to be returned.

(3) The broker performs a brokering algorithm and returns a list of candidate resource providers $\mathcal{R}^{i}$ to consumer $U_{i}$. 
(4) Consumer $U_{i}$ selects the first provider from $\mathcal{R}^{i}$ and verifies if the provider can allocate the required resources. If it can not, the consumer moves to the next provider from the list until the resources are allocated by a provider $R_{j}$.

(5) $R_{j}$ notifies the broker about the resource allocation to $U_{i}$.

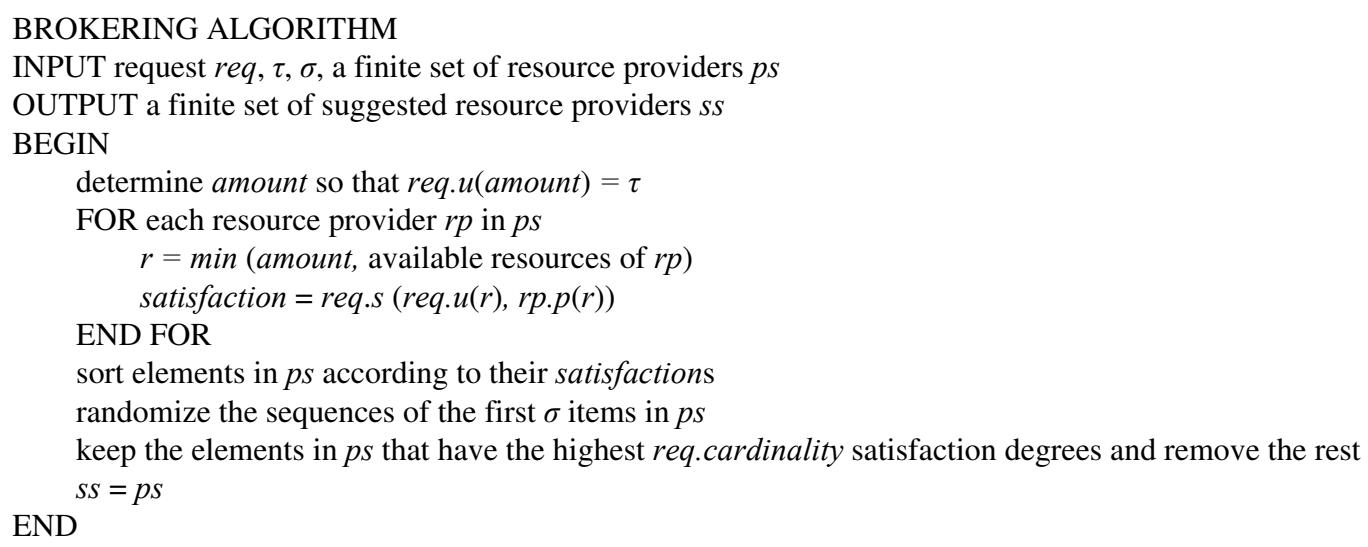

Fig. 5. The algorithm performed by the broker. The consumer request, req, is elastic. It contains the parameters describing $u$ and $\mathrm{s}$, the utility and satisfaction functions. $\tau$ is the target utility and $\sigma$ is the satisficing size. The cardinality specifies the number of resource providers to be returned by the broker.

The algorithm performed by the broker is summarized in Figure 5. The amount of resources to be allocated is determined during the algorithm according to a broker strategy. Simple strategies would be to allocate the same amount of resources to every consumer, or to allocate to every consumer a random amount of resources. A better strategy, used by our system, is to allocate an amount of resources such that the utility of each type of resource to the consumer reaches a certain target utility $\tau$. To determine the amount of resources allocated to the consumer, the broker uses Equation 13(a) derived from the definition of $u(r)$, Equation 13(b):

$$
r=e^{\frac{\ln \left(\frac{\tau}{1-\tau}\right)}{\zeta}+\ln (\omega)}(a) \quad u(r)=\frac{(r / \omega)^{\zeta}}{1+(r / \omega)^{\zeta}}
$$

Several quantities characterize the resource management policy for broker $B$ and its associ- 
ated providers and consumers:

(a) Average hourly revenue. The average is over the set of providers connected to broker $B$; the revenue of a provider is the sum of revenues from all resources it controls.

(b) Request acceptance ratio. The ratio is the number of accepted requests over the number of requests submitted by the consumers connected to broker $B$. A request is accepted if a provider able to allocate resources exists, otherwise the request is rejected and the corresponding satisfaction and utility are set to 0 .

(c) Average consumer satisfaction. The average is over the set of all consumers connected to broker $B$.

(d) Average consumer utility. This average is over the set of consumers connected to broker $B$.

In our model, a broker receives a percentage of the revenues collected by the providers connected to it. More sophisticated mechanisms are possible, for example, in addition to the percentage of the revenues collected from the providers, a broker may receive a premium from consumers based upon their level of satisfaction. This policy would encourage brokers to balance the interests of providers and consumers. Different brokers may have different policies and may be required to disclose the average values for critical parameters, such as $\tau$ and $\sigma$, and their fee structure, during the initial negotiation phase; thus, consumers and providers will have the choice to work with a broker that best matches their own objective.

\section{A Simulation Study}

Market-oriented resource allocation algorithms are very difficult to analyze analytically. To understand the behavior of the system we conducted a simulation study using YAES [9]. A 
thorough investigation would require multiple brokers, but the model is already very complex and would require additional protocols for broker selection and renegotiations so we are considering the case of a single broker.

The resource allocated by provider $R_{j}$ to consumer $U_{i}$ are represented by a resource vector $r_{i j}=\left(r_{i j}^{1} r_{i j}^{2} \ldots r_{i j}^{l}\right)$. For example, if the $k$-th component is secondary storage, then $r_{i j}^{k}=$ $20 G B$ is the amount of secondary storage provided by $R_{j}$ to consumer $U_{i}$. The associated utility and satisfaction vectors are: $u_{i j}=\left(u_{i j}^{1} u_{i j}^{2} \ldots u_{i j}^{l}\right)$ and $s_{i j}=\left(s_{i j}^{1} s_{i j}^{2} \ldots s_{i j}^{l}\right)$. The demand to capacity ratio for resource type $k$ is the ratio of the amount requested by all consumers to the total capacity of providers for resource $k, \sum_{j} c_{j}^{k}$. The level of demand is limited by the sigmoid shape of the utility curve and the finite financial resources of the consumers. In the computation of the demand-capacity ratio, for each consumer and each resource, it is assumed that for the requested $r_{i j}^{k}$ value the corresponding utility value $u_{i j}^{k}=$ 0.9 , i.e., the consumers request an amount of $r_{i j}^{k}$ that results in $u_{i j}^{k}=0.9$. The demand to capacity ratio vector for all resource types is $\eta=\left(\eta^{1} \eta^{2} \ldots \eta^{l}\right)$. For the sake of simplifying the simulation, we only consider the case when $\eta^{1}=\eta^{2}=\cdots=\eta^{l}=\eta$.

We run multiple simulation experiments for each case (50 runs/case) and compute $95 \%$ confidence intervals for the results. The parameters for our experiments are:

- $\tau$ - target utility for the consumers,

- $\sigma$ - satisficing size; reflects the choices given to the consumer by the broker, and

- $\eta$ - demand to capacity ratio; measures the commitment and, thus, the load placed upon providers.

We study the evolution in time of

- average hourly revenue,

- request acceptance ratio (the ratio of resource requests granted to total number of requests), 
- average consumer satisfaction, and

- average consumer utility.

We investigate the performance of the model for different target utilities, $\tau$, satisficing sizes, $\sigma$, and demand to capacity ratios, $\eta$. We study several scenarios, for the linear (Equation 1), EDN (Equation 5), and EDL (Equation 4) pricing strategies.

We simulate a system of 100 clusters and one broker. The number of nodes of each cluster is a random variable normally distributed with the mean of 50 and the standard deviation of 30 . Each node is characterized by a resource vector containing the CPU rate, the main memory, and the disk capacity. For example, the resource vector for a node with one $2 \mathrm{GHz} \mathrm{CPU}, 1$ GB of memory, and a $40 \mathrm{~GB}$ disk is $(2 G H z, 1 G B, 40 G B)$.

Initially, there is no consumer in the system. Consumers arrive with an inter-arrival time exponentially distributed with the mean of 2 seconds. The service time $t_{i j}$ is exponentially distributed with the mean of $\lambda$ seconds. By varying the $\lambda$ value we modify demand-capacity ratio so that we can study the behavior of the system under different loads.

The request is elastic, i.e., instead of requesting a precise amount, consumers only specify their utility and satisfaction functions. The parameters of the utility and satisfaction functions are uniformly distributed in the intervals shown in Table 2. A request provides the parameters of the utility function, $\omega$ and $\zeta$, for each element of the resource vector (CPU, Memory, Disk). We generate $\omega$ and $\zeta$ such that with a utility of 0.9 , the CPU rate, memory space, and disk space of a request are exponentially distributed with means of $2 G H z, 4 G B$, and $80 G B$, and ranges of $[0.1 G H z, 100 G H z],[0.1 G B, 200 G B]$, and $[0.1 G B, 1000 G B]$, respectively. More precisely, for each element: (a) we generate the amount $r$ according to the corresponding distribution; (b) we choose a value for $\omega$; (c) set $u=0.9$ and compute the corresponding value of $\zeta$. For a resource vector, we let the overall utility be the product of the utilities of its scalar resources, and the overall satisfaction be the product of the satisfaction for its scalar 
Table 2

The parameters for the simulation are uniformly distributed. The parameters and the corresponding intervals are shown.

\begin{tabular}{|c|c|c|c|}
\hline Parameter & CPU & Memory & Disk \\
\hline$\xi$ & {$[5,10]$} & {$[5,10]$} & {$[5,10]$} \\
$\omega$ & {$[0.4,0.9]$} & {$[0.5,1.5]$} & {$[10,30]$} \\
$\kappa$ & {$[0.02,0.04]$} & {$[0.02,0.04]$} & {$[0.02,0.04]$} \\
$\mu$ & {$[2,4]$} & {$[2,4]$} & {$[2,4]$} \\
$\epsilon$ & {$[2,4]$} & {$[2,4]$} & {$[2,4]$} \\
$\phi$ & {$[10,20]$} & {$[20,40]$} & {$[400,800]$} \\
\hline
\end{tabular}

resources.

When we study the effect of the target utility $\tau$, we use $\sigma=1$ and $\eta=1.0$; when we study the effect of $\sigma$, we use $\tau=0.9$ and $\eta=1.0$; and when we study the effect of $\eta$, we use $\tau=0.9$ and $\sigma=1$. We also compare the system performance of our scheme for several $\sigma$ values with a random strategy. In this case, we randomly choose a provider from the set of all providers, without considering the satisfaction function. To make the model more realistic, we allow a resource provider to reject a consumer's request if the available resources are insufficient to permit both satisfaction and utility to reach 0.1 .

Figures 6, 7, 8, and 9 summarize our findings. In each case, we present the three pricing strategies, linear, EDN, and EDL. The parameters for the graphs illustrating the effect of the target utility, $\tau$, at the top of the figure are: $\sigma=1, \eta=1.0$, and $\tau=0.8,0.85,0.9$, and 0.95 . The graphs illustrating the effect of the satisficing size, $\sigma$, in the middle of the figure use the following parameters: $\tau=0.9, \eta=1.0$, and $\sigma=1,10$, and 20; for the random strategy, 
$\sigma=|\mathcal{R}|=50$. The parameters for the graphs illustrating the effect of the demand to capacity ratio, $\eta$, at the bottom of the figure are: $\tau=0.9, \sigma=1$, and $\eta=0.5,1.0,1.5$, and 2.0.
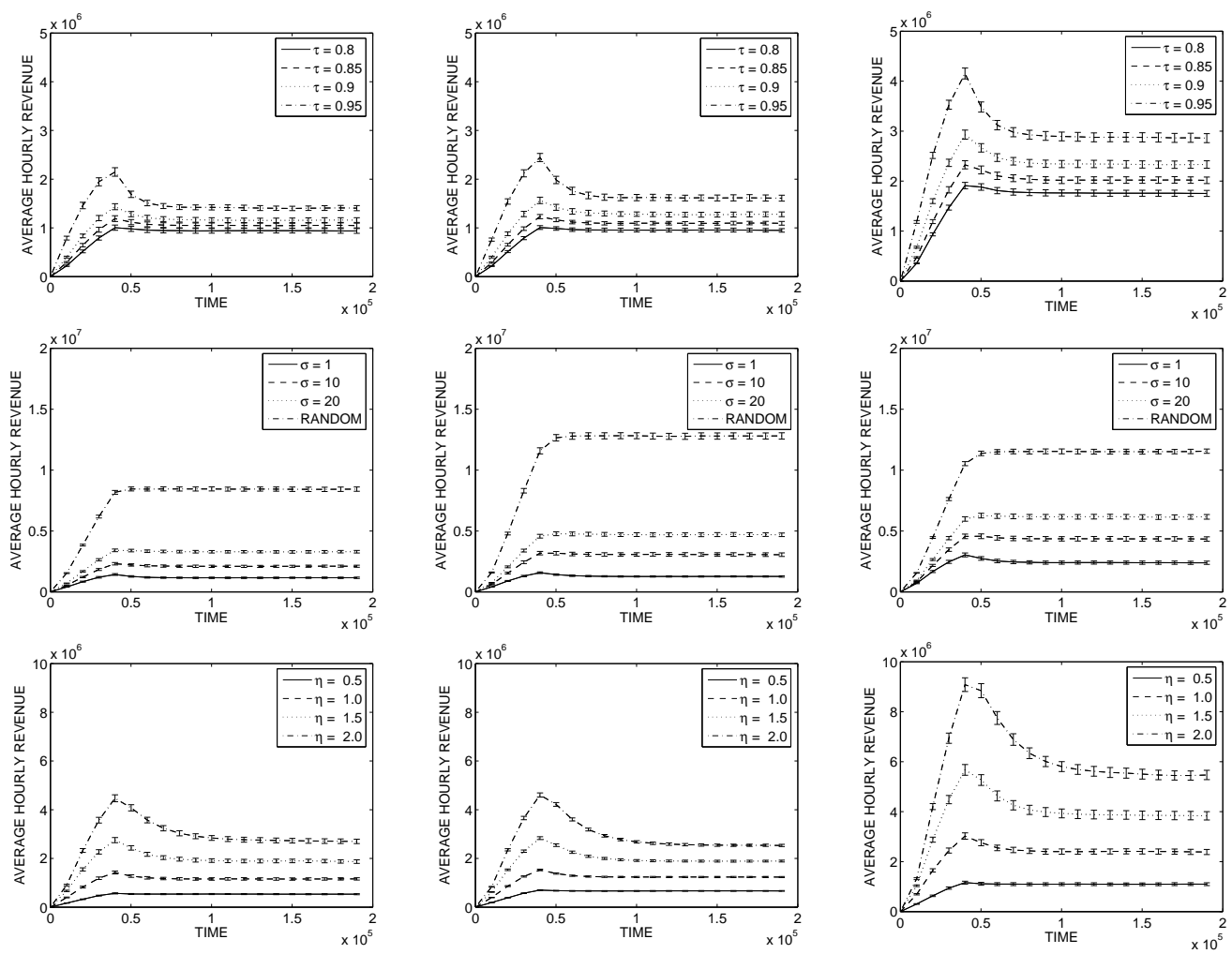

Fig. 6. Average hourly revenue vs. time (in seconds) for different target utilities, $\tau$ (top), satisficing sizes, $\sigma$ (middle), and demand to capacity ratios, $\eta$ (bottom). The three pricing strategies are: linear (left), EDN (center), and EDL (right).

The average hourly revenue is an important consideration for resource providers. We notice that the three pricing strategies exhibit similar behavior: the average hourly revenue increases rapidly during the transient period, reaches a maximum, and then converges to a steady state, as shown in Figure 6. For the same value of the target utility, $\tau$, the steady state value for the linear and the EDN pricing strategies are close to one another and almost half of those for EDL, as shown in the top row of Figure 6. In all cases, the larger $\tau$ the higher the revenue. In these simulations, $\sigma=1$ (the broker provides a single choice) and the demand to capacity ratio is $\eta=1.0$. We believe that resource fragmentation is the reason why the steady state value is lower than the maximum attained at the end of the transient period. 
Resource fragmentation is an undesirable phenomena where the amount of resources available cannot meet the target utility value for any request and resources remain idle. This effect is more pronounced for larger utility values, for example for $\tau=0.95$ the steady state value is some $20 \%$ lower than its corresponding maximum, while for $\tau=0.8$ the steady state value is close to its corresponding maximum.

The next question is if larger satisficing size affects the average revenue. A small value of $\sigma$ limits the number of choices to consumers and this restriction leads to lower average hourly revenues. In our experiments $\tau=0.9$ and $\eta=1.0$, as shown in the middle row of Figure 6. EDN and EDL are superior to linear pricing. The larger $\sigma$, the higher the average hourly revenue for the provider. The random strategy, which corresponds to the maximum value of $\sigma=|\mathcal{R}|$ leads to the highest average hourly revenue.

Lastly, we see that the demand to capacity ratio also has an impact upon the average hourly revenue that is larger for larger $\eta$ for for all three pricing strategies, as shown in the bottom row of Figure 6. The conclusion we draw from these results is that the average hourly revenue increases when we provide a higher target utility ( $\tau$ closer to 1 ), increase the satisficing size (larger $\sigma$ ), and increase the demand to capacity ratio, $\eta$, and that differential pricing strategies (EDN and EDL) are preferable to the linear one.

The request acceptance ratio for various pricing policies and choices of parameters is shown in Figure 7. We find that the request acceptance ratio shows variations during the transient period but converges to constant values in the steady state. The EDN pricing strategy appears optimal, leading to steady state values close to 1.0 for virtually every choice of parameters, except for the random $\sigma$. The steady state values for the linear and EDL strategies are also high, with values larger than 0.95 , but the exact amount is determined by the values of $\tau, \eta$ and $\sigma$. We find that the higher the values of any of these parameters, the higher the request acceptance ratio. 

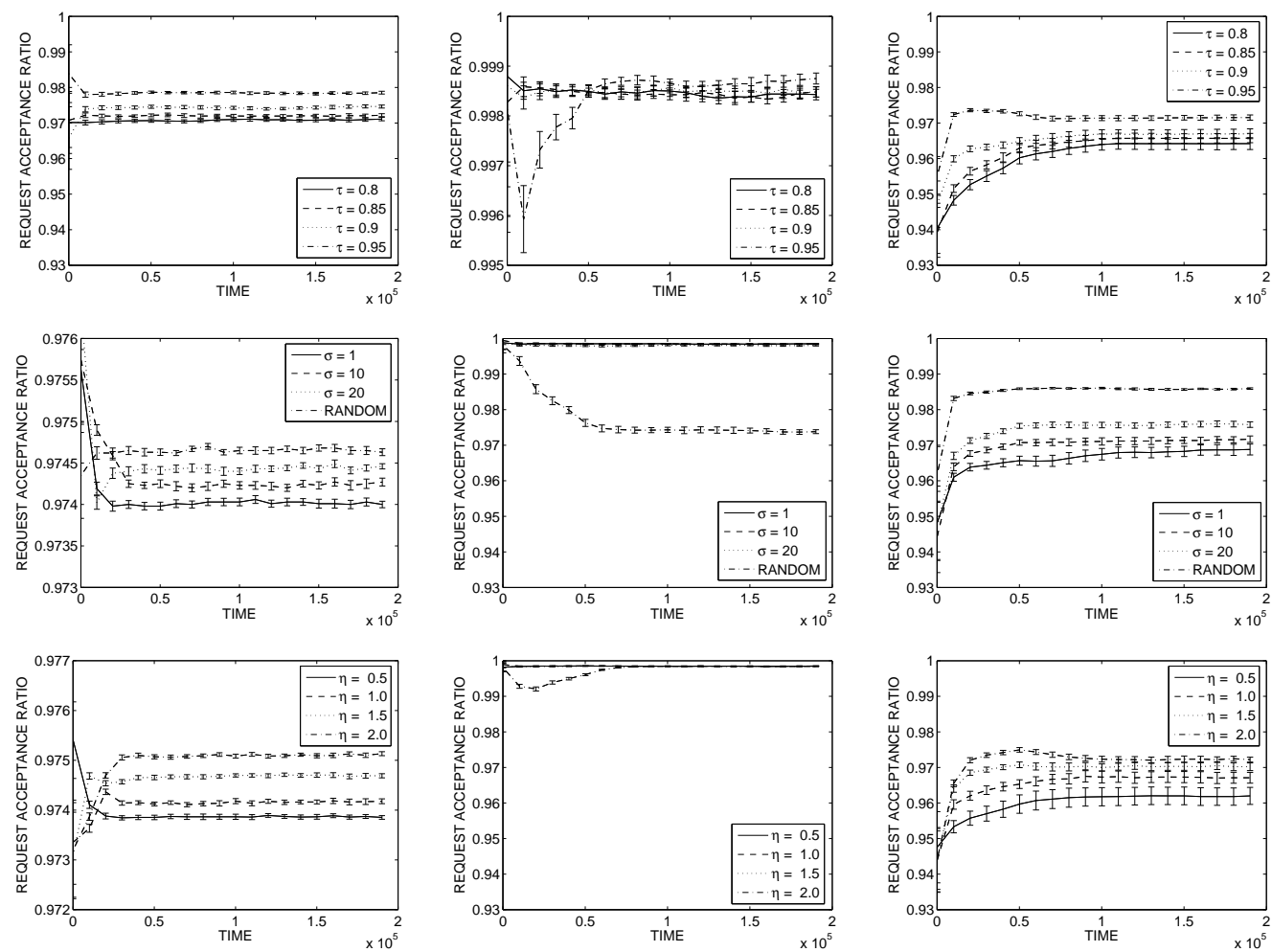

Fig. 7. Request acceptance ratio vs. time (in seconds) for different target utilities, $\tau$ (top), satisficing sizes, $\sigma$ (middle), and demand to capacity ratios, $\eta$ (bottom). The three pricing strategies are: linear (left), EDN (center), and EDL (right).

The three pricing strategies lead to very different consumer satisfaction for the same set of parameters of the simulation, even though the qualitative behavior is somehow similar in that the average consumer satisfaction decreases during the transient period and then increases and reaches a stable value in steady state, as shown in Figure 8. EDN appears to be best strategy. The larger the target utility, the lower the consumer satisfaction. The highest steady state average satisfaction is about $80 \%$ when $\tau=0.8$ and when we use the EDN strategy as compared with less than $50 \%$ for EDL and about $70 \%$ for linear pricing strategy in terms of $\sigma$. The highest satisfaction occurs when $\sigma=1$. Though this seems counterintuitive it is well justified; in this case the broker directs the consumer to that resource provider that best matches the request. When we select at random one provider from the list of all providers supplied by the broker we observe the lowest average consumer satisfaction because we 

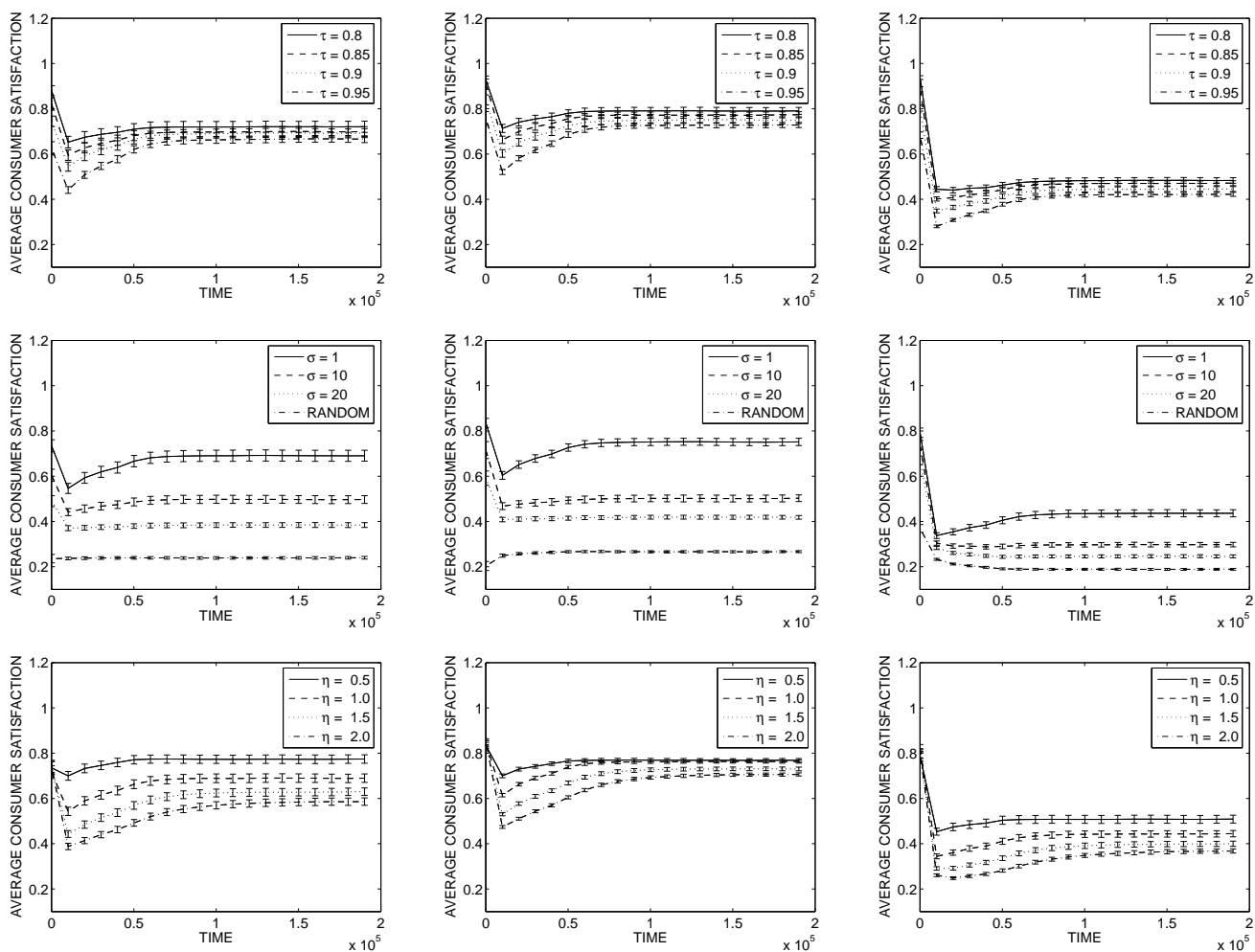

Fig. 8. Average consumer satisfaction vs. time (in seconds) for different target utilities, $\tau$ (top), satisficing sizes, $\sigma$ (middle), and demand to capacity ratio, $\eta$ (bottom). The three pricing strategies are: linear (left), EDN (center), and EDL (right).

have a high probability to select a less than optimal match for a given request. Recall that the optimal match is the top ranked element of the list of providers supplied by the broker. We also notice that a high demand to capacity ratio has a negative impact upon user satisfaction. The largest impact of the demand to capacity ratio upon the steady state average consumer satisfaction is visible for the linear pricing strategy, when the average consumer satisfaction ranges from about $55 \%$ for $\eta=2.0$ to about $75 \%$ for $\eta=0.5$.

For the same set of parameters of the simulation the three pricing strategies lead to slightly different average consumer utility values, but the qualitative behavior is similar, as shown in Figure 9. The average consumer utility decreases slowly during the transient period because of system fragmentation; some resources are allocated to consumers due to their cheaper price, although they are not enough to allow the utility to reach the target value, $\tau$. In steady 

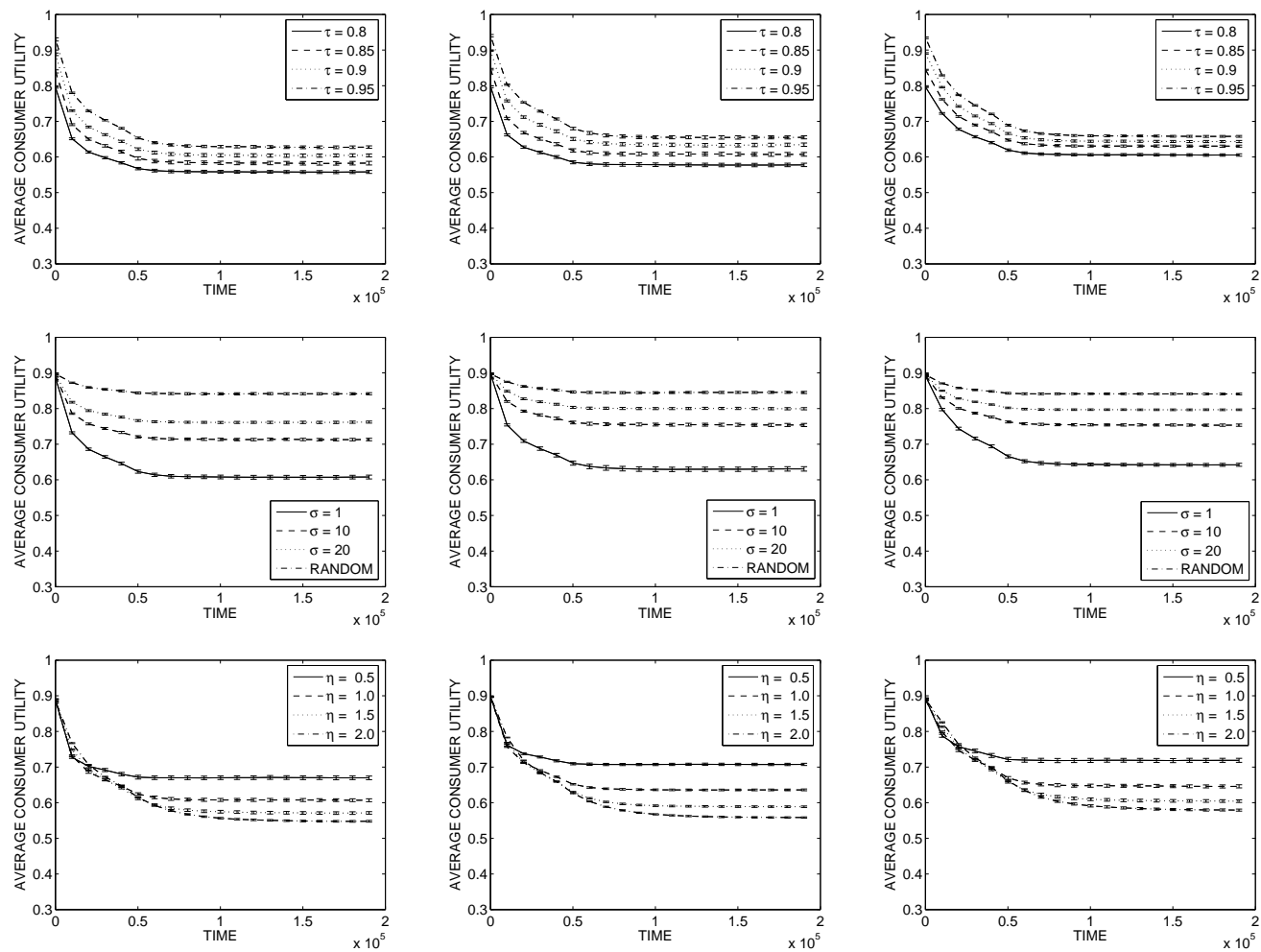

Fig. 9. Average consumer utility vs. time (in seconds) for different target utilities, $\tau$ (top), satisficing sizes, $\sigma$ (middle), and demand to capacity ratios, $\eta$ (bottom). The three pricing strategies: linear (left), EDN (center), and EDL (right).

state, the average utility reaches a stable value. Overall, the differentiated pricing strategies, EDN and EDL, perform better and reach higher steady state values. The higher the target utility, the larger the actual utility; the highest steady-sate utility is about $70 \%$ for $\tau=0.95$ for EDN and EDL, as shown in the top of Figure 9. The larger the satisficing size, the higher the actual utility; the random strategy leads to $90 \%$ utility, as shown in the middle of Figure 9. The lower the demand to capacity ratio, the higher the satisfaction.

Figure 10 summarizes the effect of the three pricing strategies upon the four quantities we monitored in our experiments, for a particular set of parameters: $\tau=0.9, \sigma=1$, and $\eta=1.0$. EDL allows the highest average hourly revenue while the linear pricing strategy leads to the lowest one, as shown in Figure 10(a). EDN leads to the highest request acceptance ratio while EDL leads to the lowest one, as shown in Figure 10(b). EDN leads to the highest consumer 


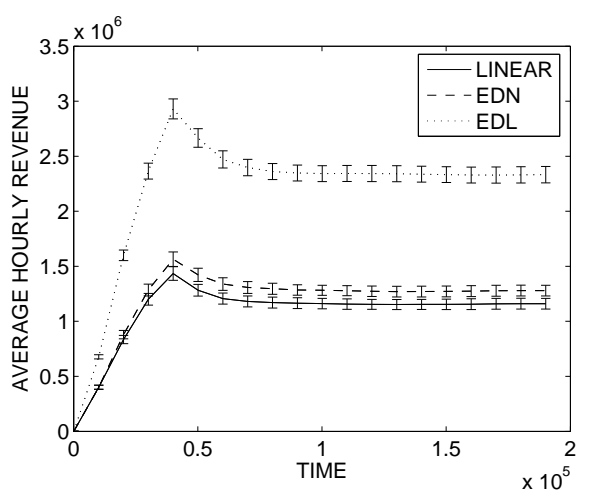

(a)

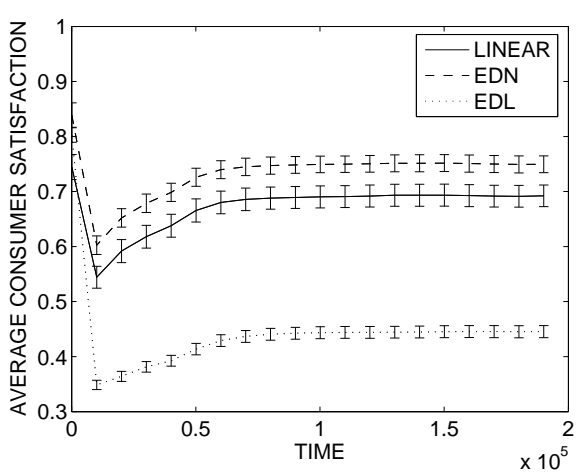

(c)

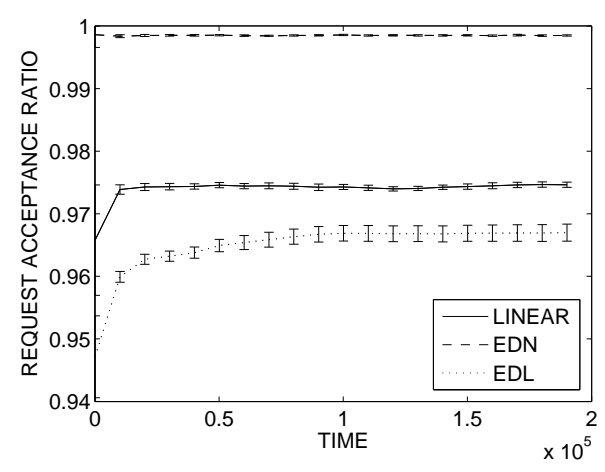

(b)

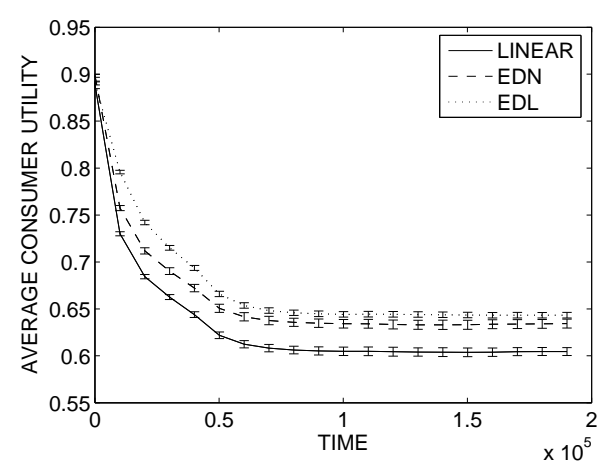

(d)

Fig. 10. (a) The average hourly revenue, (b) the request acceptance ratio, (c) the average consumer satisfaction, and (d) the average consumer utility vs. time (in seconds) for $\sigma=1, \tau=0.9$, and $\eta=1.0$, with different price functions.

satisfaction while EDL leads to the lowest one, as shown in Figure 10(c). EDL allows the highest average hourly revenue while linear pricing strategy leads to the lowest one, as shown in Figure 10(d).

\section{Conclusions and Future Work}

Economic models are notoriously difficult to study. The complexity of the utility, price, and satisfaction-based models precludes analytical studies and in this paper we report on a sim- 
ulation study. The goal of our simulation study is to validate our choice of utility, price, and satisfaction function, to study the effect of the many parameters that characterize our model, and to get some intuition regarding the transient and the steady-state behavior of our models. We are primarily interested in qualitative rather than quantitative results, i.e., we are interested in trends, rather than actual numbers.

In our model the actual shape of the utility function is controlled by the parameters dictated primarily by the application. On the other hand, the satisfaction function reflects mostly the user's constraints. The model inhibits selfish behavior: greedy consumers pay a hefty price and greedy providers who insist on high prices are avoided. The satisfaction function ensures a balance between the amount of resources consumed and the price paid for them.

The function of a broker is to monitor the system and set $\tau$ and $\sigma$ for optimal performance. For example, if the broker perceives that the average consumer utility is too low, it has two choices: increase $\tau$ or increase $\sigma$. At the same time, the system experiences an increase of the average hourly revenue and a decrease of the average consumer satisfaction. The fact that increasing utility could result in lower satisfaction seems counterintuitive, but reflects the consequences of allocating more resources; we increase the total cost possibly beyond the optimum predicated by the satisfaction function. The simulation results shown in this paper are consistent with those in $[5,6]$ where we use linear pricing and simpler models based upon a synthetic quantity to represent a vector of resources.

The EDL pricing strategy leads to the highest average consumer utility and the highest average hourly revenue, while it gives the lowest request acceptance ratio and the lowest average consumer satisfaction. The EDN pricing strategy allows the highest request acceptance ratio and the highest average consumer satisfaction, while it leads to lower average consumer utility and average hourly revenue than EDL. It is also remarkable that the average consumer satisfaction does not track the average consumer utility. This shows the importance of the 
satisfaction function.

One could argue that in practice it would be rather difficult for users to specify the parameters of their utility and satisfaction function. Of course, this is true in today's environments, but entirely feasible in intelligent environments where such information could be provided by societal services [7]. The advantages of elastic requests is likely to motivate the creation of such services in the computational economy of the future.

Even though we limit our analysis to a single broker system, we are confident that the most important conclusions we are able to draw from our model, namely that:

(i) Given a particular set of model parameters the satisfaction reaches an optimum; this value represents the perfect balance between the utility and the price paid for resources,

(ii) The satisfaction does not track the utility,

(iii) Differentiated pricing perform better than linear pricing,

(iv) Brokers can effectively control the computing economy

will still be valid for multiple broker systems. In such an environment, individual brokers could enforce different policies; providers and consumers could join the one that best matches their individual goals. The other simplifying assumptions for our analysis, e.g., the uniformity of the demand to capacity ratio for all resources available at a consumer's site, will most likely have second order effects. The restriction we impose by requiring a consumer to obtain all necessary resources from a single broker is also unlikely to significantly affect our findings.

It is very difficult to make a direct comparison between systems based on different models with different objective functions. Our results are qualitative rather than quantitative; the goal of our work is to show that our formal mathematical model captures and predicts per- 
formance trends. In Table 1 we compare the features of several systems. Performance results for existing systems are rarely reported and when they are available it would be hard to calibrate them. We are confident that a model that formalizes the selfish goals of consumers and providers, as well as societal goals, has a significant potential. Our intention is to draw the attention of the community to the potential of utility, price, and satisfaction-based resource allocation models. It is well beyond the scope of this paper to cover all angles of such a complex model.

A fair number of questions require further investigations including: (a) Are there better alternatives to the utility, price, and satisfaction functions we introduced? (b) Is the policy aiming to achieve maximum satisfaction sound, e.g., how should we take into account the societal importance of activities carried out by individual resource consumers? (c) How can we apply the models to more complex networks of resource managers? (d) What composition rules should be used to describe the utility and/or the satisfaction for a group of consumers? (e) How can we define more complex utility functions that take into account additional constraints related to system reliability and deadlines? Future work involves also the study of more complex systems including policy makers and multiple brokers.

\section{Acknowledgments}

This research was supported in part by National Science Foundation grants ACI-0296035, EIA-0296179, and CNS-0615170, the Colorado State University George T. Abell Endowment, and the DARPA Information Exploitation Office under contract No. NBCHC030137. The authors are greatly indebted to three anonymous reviewers for their constructive comments which helped improve the quality of this paper. One of the authors (dcm) acknowledges useful discussions regarding the Kelly model with Don Towsley. Preliminary versions and performance results presented in this paper were reported in $[5,6]$. 


\section{References}

[1] Y. Amir, B. Awerbuch, A. Barak, R. S. Borgstrom, and A. Keren. An opportunity cost approach for job assignment in a scalable computing cluster. IEEE Trans. on Parallel and Distributed Systems, 11(7):760-768, 2000.

[2] Y. Amir, B. A. B, and R.S.Borgstrom. A cost-benefit framework for online management of a metacomputing system. In Proc. 1st Int. Conf. on Information and Computation Economies (ICE 98), pp. 14-47. ACM Press: New York, 1998.

[3] A. Anastasiadi, S. Kapidakis, C. Nikolaou, and J. Sairamesh. A computational economy for dynamic load balancing and data replication. In Proc 1st Int. Conf. on Information and Computation Economics ICE'98, 1998.

[4] L. Badia and M. Zorzi. On utility-based radio resource management with and without service guarantees. In Proc. ACM MSWiM 2004, Modelling, Analysis, and Simulation of Wireless and Mobile Systems, pp. 244-251. ACM Press, 2004.

[5] X. Bai, L. Bölöni, D. C. Marinescu, H. J. Siegel, R. A. Daley, and I.-J. Wang. Are utility, price, and satisfaction based resource allocation models suitable for large-scale distributed systems? In Proc. 3rd Int. Workshop on Grid Economics and Business Models (GECON 2006), Singapore, 2006.

[6] X. Bai, L. Bölöni, D. C. Marinescu, H. J. Siegel, R. A. Daley, and I.-J. Wang. A brokering framework for large-scale heterogeneous systems. 15th Heterogeneous Computing Workshop (HCW 2006) in Proc. 20th IEEE Int. Parallel and Distributed Processing Symp. (IPDPS 2006) (CD Proceedings), Rhodes, 2006.

[7] X. Bai, H. Yu, G. Wang, Y. Ji, G. M. Marinescu, D. C. Marinescu, and L. Bölöni. Coordination in intelligent grid environments. Proc. of the IEEE, 93(3):613-630, 2005.

[8] M. Blaugh. Economic Theory in Retrospect. Cambridge University Press, 5th Edition, ISBN 978-0521577014, 1997 
[9] L. Bölöni and D. Turgut. YAES - a modular simulator for mobile networks. In Proc. 8-th ACM/IEEE Int. Symp. on Modeling, Analysis and Simulation of Wireless and Mobile Systems MSWIM 2005, pp. 169-173, 2005.

[10] R. Buyya, D. Abramson, and J. Giddy. Nimrod/g: An architecture of a resource management and scheduling system in a global computational grid. In Proc. 4 th Int. Conf. on High Performance Computing in the Asia Pacific Region, 1:283-289, 2001.

[11] R. Buyya, H. Stockinger, J. Giddy, and D. Abramson. Economic models for management of resources in peer-to-peer and grid computing. In Proc. SPIE Int. Conf. on Commercial Applications for High-Performance Computing, pp. 13-25, Denver, 2001.

[12] B. Chun and D. Culler. Market-based proportional resource sharing for clusters. Technical report, UC Berkeley, Sept. 1999.

[13] CONDOR. URL http://www.cs.wisc.edu/condor/.

[14] B. F. Cooper and H. Garcia-Molina. Peer-to-peer data preservation through storage auctions. IEEE Trans. Parallel Distrib. Syst., 16(3):246-257, 2005.

[15] ContractNet FIPA standard URL http://www.fipa.org/specs/fipa00029/

[16] R. Frisch. Propagation problems and impulse problems in dynamc economics. In Econnomic Essays in Honour of Gustav Cassel, pp. 691-704, Allen and Unwin, London, 1933. Reprinted in "Readings in Business Cycles," R. A. Gordon and L. R. Klein, Eds., 1966.

[17] P. Ghosh, N. Roy, S. K. Das, and K. Basu. A pricing strategy for job allocation in mobile grids using a non-cooperative bargaining theory framework. J. Parallel Distrib. Comput., 65(11):1366-1383, 2005.

[18] J. Gomoluch and M. Schroeder. Market-based resource allocation for grid computing: A model and simulation. In Int. Middleware Conf., Workshop Proc., pp. 211-218, Rio de Janeiro, 2003.

[19] C. Grothoff. Resource allocation in peer-to-peer networks - an excess-based economic model. Wirtschaftsinformatik, 45(3):285-292, 2003. 
[20] L. He and T. R. Ioerger. Task-oriented computational economic-based distributed resource allocation mechanisms for computational grids. In Proc. Int. Conf. on Artificial Intelligence, volume 1, pp. 462-468, Las Vegas, 2004.

[21] G. Heiser, F. Lam, and S. Russel. Resource management in the Mungi single-address-space operating system. In Proc. 21 st Australasian Computer Science Conf., pp. 417-428, 1998.

[22] HP Grid computing. URL http://www.hp.com/techservers/grid.

[23] IBM Grid computing. URL http://www.ibm.com/grid.

[24] S. U. Khan and I. Ahmad. Non-cooperative, semi-cooperative, and cooperative games-based grid resource allocation. Proc. Int. Parallel \& Distrib. Proc. Symp, (IPDPS 2006), 2006.

[25] F. Kelly. Models for a self-managed Internet. Philosophical Transactions of the Royal Society, A358:2335-2348, 2000.

[26] S. Lalis and A. Karipidis. Jaws: An open market-based framework for distributed computing over the internet. In R. Buyya and M. Baker, Eds., GRID, volume 1971 of LNCS, Springer, pp. 36-46. 2000.

[27] Mariposa. URL http://mariposa.cs.berkeley.edu/.

[28] L. W. McKnight and J. Boroumand. Pricing Internet services: Approaches and challenges. IEEE Computer, 33(2):128-129, 2000.

[29] N. Nisan, S. London, O. Regev, and N. Camiel. Globally distributed computation over the internet - the popcorn project. In ICDCS '98: Proc. 18th Int. Conf. on Distributed Computing Systems, pp. 592-601, Washington, 1998. IEEE Computer Society.

[30] Nobel Lectures, Economics 1969-1980, From Nobel Lectures, Economics 1969-1980, A. Lindbeck, Editor, World Scientific Publishing, Singapore, 1992.

[31] D. Reed, I. Pratt, P. Menage, S. Early, and N. Stratford. Xenoservers: Accountable execution of untrusted programs. In Workshop on Hot Topics in Operating Systems, pp. 136-141, 1999. 
[32] SETI@home. URL http://setiathome.ssl.berkeley.edu/.

[33] W. Shen, Y. Li, H. H. Genniwa, and C. Wang. Adaptive negotiation for agent-based grid computing. Journal of the American Statistical Association, 97(457), 2002.

[34] H. A. Simon. Models of Man. Wiley, 1957.

[35] R. G. Smith. The contract net protocol: high-level communication and control in a distributed problem solver. IEEE Trans. on Computers Vol C-29, 12:1104 - 1113, 1980.

[36] B. Snowdown, H. R. Vane. Modern Macroeconomic: Tts Origin, Development and Current Status. Edward Elgar Publishing, New Edition, ISBN 978-1845422080, 2005.

[37] M. Stonebraker, R. Devine, M. Kornacker, W. Litwin, A. Pfeffer, A. Sah, and C. Staelin. An economic paradigm for query processing and data migration in Mariposa. In Proc. 3rd Int. Conf. on Parallel and Distributed Information Systems, 1994.

[38] Sun Microsystems utility computing. URL http://www.sun.com/service/utility.

[39] H. R. Varian. Intermediate Microeconomics: A Modern Approach. Norton, New York, 1999.

[40] W. Vickrey. Counterspeculation and competitive sealed tenders. Journal of Finance, 16(1):8-37, 1961.

[41] C. A. Waldspurger, T. Hogg, B. A. Huberman, J. O. Kephart, and W. S. Stornetta. Spawn: A distributed computational economy. Software Engineering, 18(2):103-117, 1992.

[42] R. Wolski, J. S. Plank, J. Brevik, and T. Bryan. Analyzing market-based resource allocation strategies for the computational Grid. The Int. Journal of High Performance Computing Applications, 15(3):258-281, 2001.

[43] R. Wolski, J. S. Plank, J. Brevik, and T. Bryan. G-commerce: Market formulations controlling resource allocation on the computational grid. In Proc. 15th Int. Parallel \& Distributed Processing Symposium (IPDPS-01), pp. 23-27, San Francisco, CA, April 2001. 